\title{
Bounding the Number of Connected Components of a Real Algebraic Set
}

\author{
Riccardo Benedetti, ${ }^{1}$ Francois Loeser, ${ }^{2}$ and Jean Jacques Risler ${ }^{3}$ \\ ${ }^{1}$ Dipartimento di Matematica, Universita di Pisa, Italia \\ ${ }^{2}$ Centre de Mathématiques, Ecole Polytechnique, Palaiseau, France \\ ${ }^{3}$ Universite Paris VI, ENS, Paris, France
}

\begin{abstract}
For every polynomial map $f=\left(f_{1}, \ldots, f_{k}\right): \mathbb{R}^{n} \rightarrow \mathbb{R}^{k}$, we consider the number of connected components of its zero set, $B\left(Z_{f}\right)$, and two natural "measures of the complexity of $f$," that is the triple $(n, k, d), d$ being equal to $\max$ (degree of $f_{i}$ ), and the $k$-tuple $\left(\Delta_{1}, \ldots, \Delta_{k}\right), \Delta_{i}$ being the Newton polyhedron of $f_{i}$, respectively. Our aim is to bound $B\left(Z_{f}\right)$ by recursive functions of these measures of complexity. In particular, with respect to $(n, k, d)$ we shall improve the well-known Milnor-Thom's bound $\mu_{d}(n)=d(2 d-1)^{n-1}$. Considered as a polynomial in $d, \mu_{d}(n)$ has leading coefficient equal to $2^{n-1}$. We obtain a bound depending on $n, d$, and $k$ such that if $n$ is sufficiently larger than $k$, then it improves $\mu_{d}(n)$ for every $d$. In particular, it is asymptotically equal to $\frac{1}{2}(k+1) n^{k-1} d^{n}$, if $k$ is fixed and $n$ tends to infinity. The two bounds are obtained by a similar technique involving a slight modification of Milnor-Thom's argument, Smith's theory, and information about the sum of Betti numbers of complex complete intersections.
\end{abstract}

\section{Introduction}

Consider any polynomial map $f=\left(f_{1}, \ldots, f_{k}\right): \mathbb{R}^{n} \rightarrow \mathbb{R}^{k}$. Given any suitable "measure of the complexity" of $f$, it is of primary interest (also in view of applications) to obtain explicit bounds for the number $B\left(Z_{f}\right)$ of connected components of the zero set of $f, Z_{f}=\left\{x \in \mathbb{R}^{n} \mid f(x)=0\right\}$. A basic result of this kind is the well-known Milnor-Thom's bound in terms of the triple $(n, k, d)$, where

$$
d=\max \left\{\text { degree of } f_{i}, i=1, \ldots, k\right\} .
$$

Actually Milnor-Thom's bound

$$
\mu_{d}(n)=d(2 d-1)^{n-1}
$$


depends only on $(n, d)$. Another measure of complexity is the so-called additive complexity. Milnor-Thom's argument applies with minor changes to this case, producing recursive bounds (see [R1] or [BR] for details).

A further example of the natural measure of complexity is given by the $k$-tuple of the Newton polyhedra (see the definition later) of $f_{1}, \ldots, f_{k}$ : in some sense they involve the triple $(n, k, d)$ in a "structured way."

The aim of this paper is to construct, in a recursive way, two bounds for $B\left(Z_{f}\right)$ in terms of $(n, k, d)$ and of the Newton polyhedra, respectively (actually the second bound will be a function of the mixed volumes of a finite family of polyhedra constructed by beginning from the Newton polyhedra). In some cases the first bound improves the one by Milnor and Thom, in a sense made precise later. These bounds are constructed by a similar technique based on a very simple ideas, as is shown in the following sketch.

\section{An Outline of the Construction of the Bounds for $B\left(Z_{f}\right)$}

Assume for simplicity that $Z_{f}$ is compact (the noncompact case can be reduced to a question in lower dimensions, so we can work by induction). In Milnor-Thom's proofs the problem of bounding $B\left(Z_{f}\right)$ is reduced to the problem of bounding the number of connected components of the set $f^{-1}\left(\partial_{B}\right)$, where $B$ is a small "generic" ball surrounding the origin. Here, in order to get the bound in terms of $(n, k, d)$, we replace the ball by a small generic simplex $\Delta$. The advantage of the simplex is that it does not increase the degree. Call a connected component of $f^{-1}(\partial \Delta)$ pure with respect to a fixed face $\sigma$ of $\Delta$ if its range intersects $\sigma$ but none of its subfaces. We only need to bound the number of pure components for every face of $\partial \Delta$, hence it is enough to bound, for every $\sigma$, the number of compact components of $S=f^{-1}\left(H_{\sigma}\right)$, where $H_{\sigma}$ is the affine space spanned by $\sigma$. By genericity, $S$ is nonsingular. We consider the projective closure $S^{\prime}$ of $S$ in $\mathbb{R} P^{n} \subset \mathbb{C} P^{n}$, and approximate $S^{\prime}$ by a complex smooth projective complete intersection (Bertini theorem). Then we are able to finish by using known formulas for the sum of the Betti numbers of complex complete intersections (in terms of $n, k$, and $d$ ) and Smith's theory. To get the bound in terms of Newton polyhedra we use the same construction, but replacing the simplex by a small generic rectangular parallelepiped (because it is more convenient to control the Newton polyhedra) and finally use formulas by Hovansky for the Euler characteristic of complex complete intersections in terms of Newton polyhedra.

Note that the use of Smith's theory in this kind of question has already been suggested at the end of [T].

We also use some basic facts about real algebraic and semialgebraic sets. Whenever no explicit reference is given, the reader may check [BCR] or [BR]. We thank the referees for useful suggestions to the final version of this paper. The paper is structured as follows: first we obtain the bound in terms of $(n, k, d)$ and secondly we obtain the one for the Newton polyhedra. Finally, we consider some examples in order to compare the bounds obtained. 


\section{Bounding $B\left(Z_{f}\right)$ in Terms of $(n, k, d)$}

Let $A(d, n, k)$ be the set of polynomial maps $f=\left(f_{1}, \ldots, f_{k}\right): \mathbb{R}^{n} \rightarrow \mathbb{R}^{k}$, such that, for every $i=1, \ldots, k, f_{i} \in \mathbb{R}\left[X_{1}, \ldots, X_{n}\right]$ and the degree of $f_{i}$ is less or equal to $d$. For every topological space $X, B(X)$ denotes the number of connected components of $X$. For $f \in A(d, n, k), Z_{f}=\left\{x \in \mathbb{R}^{n} \mid f(x)=0\right\}$. Set

$$
\Phi_{d}(n, k)=\sup \left\{B\left(Z_{f}\right) \mid f \in A(d, n, k)\right\} .
$$

As we have recalled, Milnor-Thom's results state [M1], [T]

$$
\Phi_{d}(n,, k) \leq \mu_{d}(n)=d(2 d-1)^{n-1} .
$$

\section{A. Remarks on $\Phi_{d}(n, k)$}

1. Actually, the above inequality is a corollary of a stronger fact: $\mu_{d}(n)$ gives a bound for the sum of all Betti numbers of $Z_{f}$, over an arbitrary field of coefficients - for instance $\mathbb{Z} / 2 \mathbb{Z}$. (The definition of Betti numbers needs the machinery of algebraic topology which we cannot include here. We refer to any wellestablished text of (elementary) algebraic topology for this definition. Suffice it to say that every such Betti number is the dimension of a suitable finite-dimensional vector space on the field of coefficients, associated to $Z_{f}$ by a geometric-algebraic construction, and that - this is important to the aim of this paper - the first Betti number is exactly the number of connected components of $Z_{f}$.) On the other hand, for several applications (for example, producing lower bounds in complexity theory, as in [B1], or computing the number of polytope types, as in [GP]), it is the number of connected components which has actually been used.

2. $\mu_{d}(n)$ is a polynomial function of $d$ of degree $n$, with leading coefficient $2^{n-1}$; thus it is exponential in $n$. Note that

$$
\Phi_{d}(n, k) \geq(d / 2)^{n}
$$

To see this, it is enough to consider $P=\Sigma_{1 \leq j \leq n}\left(\left(X_{j}-1\right) \cdots\left(\left(X_{j}-d\right)\right)^{2}\right.$ and take $f_{1}=\cdots=f_{k}=P$. Thus $n$ is the best degree we can reasonably expect for any bound polynomial in $d$. Roughly speaking, we are going to obtain a bound polynomial in $d$ of degree $n$, with leading coefficient which is polynomial in $n$, when $k$ is fixed.

3. Note that $\mu_{d}(n)$ does not depend on $k$. This a consequence of the trivial remark that $\Phi_{d}(n, k) \leq \Phi_{2 d}(n, 1)$, obtained by taking $F=f_{1}^{2}+\cdots+f_{k}^{2}$ and noticing that $Z_{f}=Z_{F}$. The proof in [M1] actually bounds $B\left(Z_{F}\right)$ by $\mu_{d}(n)$. We want to distinguish the roles of $n$ and $k$ and obtain a bound such that if $n$ is sufficiently larger than $k$, then it improves $\mu_{d}(n)$ for every $d$. One of the referees suggested to us that, with a fairly slight modification of Milnor and Thom's methods, we obtain the bound

$$
\Phi_{d}(n, k) \leq d^{(k+n-1)}
$$


(We sketch how to arrive at it: The proof in [M1] ends by bounding the number of nondegenerate solutions of the system

$$
\left\{\begin{array}{l}
g=\left(\sum_{1 \leq i \leq k} f_{1}^{2}\right)+e^{2}\left(\sum_{1 \leq j \leq n} x_{j}^{2}\right)=t^{2} \\
\partial g / \partial x_{j}=0, \quad j=1, \ldots, n
\end{array}\right.
$$

where $e$ and $t$ are suitable constants. We consider an equivalent system involving new variables $y_{i}$ :

$$
\left\{\begin{array}{l}
f_{i}=y_{i}, \quad i=1, \ldots, k \\
\left(\sum_{i} \partial f_{i} / \partial x_{s}\right)+2 e^{2} x_{s}=0, \quad s=2, \ldots, n \\
\left(\sum_{i} y_{i}^{2}\right)+e^{2}\left(\sum_{j} x_{j}^{2}\right)=t^{2}
\end{array}\right.
$$

Using Bezout's theorem in order to bound the nondegenerate solutions of the last system, we obtain $2 d^{(n+k-1)}$ and, hence, arguing as in [M1], the bound $d^{(n+k-1)}$ for the number of connected components.)

It is easy to see that for every $(d, k)$ there exists $n_{0}=n_{0}(d, k)$ such that if $n \geq n_{0}$, then $d^{(n+k-1)}$ improves $\mu_{d}(n)$-for instance it should work if $n \geq k \log (d)-$; a similar fact holds with respect to the bound we obtain. On the other hand, it gives the best degree in $d$ only for $k=1$. In this case we get

$$
\Phi_{d}(n, 1) \leq d^{n}
$$

But we can see that for every $n$ there exists $d_{n}$ such that if $d \geq d_{n}$, then our bound for $\Phi_{d}(n, 1)$ is better than $d^{n}$.

In $[\mathrm{W}]$ we could find

$$
\Phi_{d}(n, 1) \leq 2 d^{n}
$$

obtained by a method rather different with respect to Milnor and Thom and having some analogy with the one used in this paper.

4. For $\Phi_{d}(2,1)$ we know the sharp bound $\left(d^{2}-d+2\right) / 2$ (see $[\mathrm{BR}]$ ).

5. Note that $\Phi_{d}(n, k)$ is trivially a nondecreasing function of $k$. In fact, if $\left(f_{1}, \ldots, f_{k}\right) \in A(d, n, k)$, then $\left(f_{1}, \ldots, f_{k}, f_{k}\right) \in A(d, n, k+1)$ and they have the same zero set.

We remark that $\Phi_{d}(n, n)$ is the "worst" case. That is

Proposition 2.1. For every $k \geq n, \Phi_{d}(n, k)=\Phi_{d}(n, n)$.

Proof. It is enough to show that for every $f \in A(d, n, k), k>n$, there exists $g \in A(d, n, k-1)$ such that $B\left(Z_{f}\right) \leq B\left(Z_{g}\right)$. Set $E=f\left(\mathbb{R}^{n}\right)$. $E$ is a semialgebraic set in $\mathbb{R}^{k}$ of dimension $r \leq n \leq k$. Consider a straight line through the origin of $\mathbb{R}^{k}$ in 
general position with respect to $E$. This means the following: we may stratify $E$ by a finite number of analytic semialgebraic submanifolds $M_{i}$ (not necessarily closed) of $\mathbb{R}^{k}$ such that $E$ is the disjoint union of the $M_{i}$ 's-called the strata of the stratification-and the stratification is "good" if it is a Whitney stratification. (This notion is a little technical and we cannot review it completely. We refer, for example, to [GWDL] or to [BCR] for the exact definition. It suffices to recall the main properties (a) if $M_{i}$ is a stratum, then its closure in $\mathbb{R}^{k}$ is union of strata; (b) if $M_{j} \neq M_{i}$ is in the closure of $M_{i}$, then $\operatorname{dim} M_{j}<\operatorname{dim} M_{i}$; (c) (this is roughly the Whitney condition) if $M_{i}$ and $M_{j}$ are as in (b), $x \in M_{j}, y \in M_{i}$, let us denote $T M_{i y}$ as the tangent space to $M_{i}$ at $y ; y$ is identified with the origin of $T M_{i y}$, and $t(x, y)$ is the straight line passing through $x$ and $y$. Then if $y$ is "close" to $x, t(x, y)$ is "almost contained" in $T M_{i y}$. This condition is very useful in transversality arguments like the one below, for the following reason: if $x, M_{i}, M_{j}$ are as in (c) and a manifold $N$ is transverse to $M_{j}$ at $x$, then there exists a neighborhood $U$ of $x$ in $\mathbb{R}^{k}$ such that $N$ is transverse to $U \cap M_{i}$.)

If $0 \notin E$, the $L$ is in general position if it is transverse to every $M_{i}$. If $0 \in E$ and if $M_{0}$ is the stratum containing 0 , then $L$ is in general position if it is transverse both to $M_{0}-\{0\}$ and to every $M_{i}, i \neq 0$, and $L \cap\left(T M_{00}\right)=\{0\}$. In the first case, standard transversality arguments show that the subset of $\mathbb{R} P^{k-1}$ (which is the space of lines through the origin of $\mathbb{R}^{k}$ ) of lines in general position with respect to $E$ is an open dense (semialgebraic) subset (see, for instance, [GWDL]).

A little more care is necessary when $0 \in E$ in order to get the transversality conditions. Assume that $M_{0}$ is contained in the closure of $M_{i}$. Consider $\Gamma_{i} \subset M_{i} \times$ $\mathbb{R} P^{k-1}$ defined by $(p, L) \in \Gamma_{i}$ if $p \in M_{i}$ and $L$ is the line through 0 and $p$. Both $\Gamma_{i}$ and its closure $\Gamma_{i}^{\prime}$ are semialgebraic sets of dimension $m_{i}<k$. If $\pi_{1}$ and $\pi_{2}$ are the projections of $\Gamma_{i}^{\prime}$ on $E$ and $\mathbb{R} P^{k-1}$, respectively, then $\pi_{2}\left(\pi_{1}^{-1}(0)\right)$ is a semialgebraic set of dimension $<m_{i}$. Using this fact it is easy to conclude that also in this case the lines in general position make a dense set. Note that if $L$ is in general position, then 0 is isolated in $L \cap E$. Let $L$ be such a line; we assume that it is defined by $k-1$ equations of degree $1: h_{f}\left(y_{1}, \ldots, y_{k}\right)=0, j=1, \ldots, k-1$. Then $g=(h \circ f)$, $h=\left(h_{1}, \ldots, h_{k-1}\right)$, belongs to $A(d, n, k-1)$ and $z_{g}=z_{f} \cup f^{-1}(L \cap(E-\{0\})$ (it is a disjoint union). Hence $B\left(Z_{g}\right) \geqq B\left(Z_{f}\right)$.

Remark 2.2. It is easy to deduce from the above proof that there exists a generic $k-n$ plane $H$ through the origin of $\mathbb{R}^{k}$, defined by $n$ equations of degree $1: h_{1}=\cdots=$ $h_{n}=0$, such that $g=(h \circ f) \in A(d, n, n)$ and $Z_{g}=Z_{f} \cup\left\{p_{1}, \ldots, p_{s}\right\}$ where the $p_{i}$ 's are isolated points corresponding to the transverse intersection of $H$ with the strata of dimension $n$ of $E$.

It is also clear that we have actually proved a more precise fact; let us denote by $\operatorname{Betti}_{F}\left(Z_{f}\right)$ the sum of the Betti numbers of $Z_{f}$ over the field $F$, and $\operatorname{Betti}_{F}(d, n, k)=$ $\sup \left\{\operatorname{Betti}_{F}\left(Z_{f}\right) \mid f \in A(d, n, k)\right\}$. Then we have

Proposition 2.3. For every $k \geqq n, \operatorname{Betti}_{F}(d, n, k)=\operatorname{Betti}_{F}(d, n, n)$.

Remark 2.4. For the crucial case $A(d, n, n)$ we do not know any example with $B\left(Z_{f}\right)$ bigger than $d^{n}$. 


\section{B. Recursive Definition of a Bound for $\Phi_{d}(n, k)$}

In this section we give the recursive definition of an upper bound for $\Phi_{d}(n, k)$ depending on the triple $(d, n, k)$. Later we show some property of the bound obtained, noticing, in particular, how it improves Milnor-Thom's bound.

Let us first introduce some further notation. For every topological space $X$, $\mathrm{B}^{\mathrm{c}}(X)$ denotes the number of compact connected components of $X$. Then set

$$
\phi_{d}^{\mathrm{c}}(n, k)=\sup \left\{B^{\mathfrak{c}}\left(Z_{f}\right) \mid f \in A(d, n, k)\right\} .
$$

For $n \geq k, \mathrm{C}(\mathrm{d}, \mathrm{n}, \mathrm{k})$ denotes the set of smooth complex complete intersections in $\mathbb{C} P^{n}$ defined by $k$ real homogeneous polynomials of degree at most $d$. Set

$$
\theta_{k}^{n}(d)=\sup \left\{B\left(Y \cap \mathbb{R} P^{n}\right) \mid Y \in C(d, n, k)\right\} .
$$

It is well known (see, for instance, [F] and [R2]) that the topology of $Y \in C(d, n, k)$ depends only on $d_{1}, \ldots, d_{k}, n, k$ (if $Y=\left\{g_{1}=\cdots=g_{k}=0\right\}$ and the degree of $g_{i}$ is equal to $d_{i}$ ). then

In particular, if $\operatorname{Betti}(Y)$ denotes the sum of the Betti numbers of $Y$ over $\mathbb{Z} / \mathbb{Z}$,

$$
\operatorname{Betti}(Y)=H_{k}^{n}\left(d_{1}, \ldots, d_{k}\right),
$$

where the last term is recursively defined as follows:

$$
\begin{gathered}
H_{k}^{n}\left(d_{1}, \ldots, d_{k}\right)= \begin{cases}\chi_{k}^{n}\left(d_{1}, \ldots, d_{k}\right) & \text { if }(n-k) \text { is even, } \\
2(n-k+1)-\chi_{k}^{n}\left(d_{1}, \ldots, d_{k}\right) & \text { if }(n-k) \text { is odd, }\end{cases} \\
\begin{cases}\chi_{k}^{n}\left(d_{1}, \ldots, d_{k}\right)= \begin{cases}n+1 & \text { if } k=0, \\
d_{1} d_{2}, \ldots, d_{k} & \text { if } k=n,\end{cases} \\
\chi_{k}^{n}\left(d_{1}, \ldots, d_{k}\right)=d_{k} \chi_{k-1}^{n-1}\left(d_{1}, \ldots, d_{k-1}\right)-\left(d_{k}-1\right) \chi_{k}^{n-1}\left(d_{1}, \ldots, d_{k}\right) & \text { if } 0<k<n .\end{cases}
\end{gathered}
$$

Note that if $d_{i} \leqq d$ for every $i$, then

$$
H_{k}^{n}\left(d_{1}, \ldots, d_{k}\right) \leqq H_{k}^{n}(d)
$$

where $H_{k}^{n}(d)=H_{k}^{n}(d, \ldots, d)$.

Our bound is actually defined by the following proposition.

Proposition 2.5. Let $n>k$, then:

$$
\begin{aligned}
\theta_{k}^{n}(d) & \leq \frac{1}{2} H_{k}^{n}(d), \\
\Phi_{d}(n, k) & \leq \Phi_{d}^{c}(n, k)+2 \Phi_{d}(n-1, k), \\
\Phi_{d}^{c}(n, k) & \leq \sum_{1 \leq j \leq k}\left(\begin{array}{c}
k+1 \\
j
\end{array}\right) \theta_{k+1-j}^{n}(d) .
\end{aligned}
$$


Assuming the above proposition we can define our bound for $\Phi_{d}(n, k)$ as follows:

if $k \geq n$ set $\lambda(d, n, k)=\mu_{d}(n)$ (Milnor-Thom),

if $k<n$ we apply $(n-k)$ times the proposition obtaining $\lambda(d, n, k)$ such that, obviously, $\Phi_{d}(n, k) \leq \lambda(d, n, k)$.

That is,

$$
\begin{aligned}
\lambda(d, n, k)= & \frac{1}{2} \sum_{1 \leq j<k}\left(\begin{array}{c}
k+1 \\
j
\end{array}\right) \\
& \times\left(H_{k+1-j}^{n}(d)+2 H_{k+1-j}^{n-1}(d)+\cdots+2^{n-k-1} H_{k+1-j}^{k+1}(d)\right)+2^{n-k} \mu_{d}(k) .
\end{aligned}
$$

It is clear that $\lambda(d, n, k)$ is an upper bound for $\Phi_{d}(n, k)$. Before proving Proposition 2.5 , we note some properties of this bound, showing, in particular, how it improves $\mu_{d}(n)$ or $d^{n+k-1}$.

Proposition 2.6. We have

$$
\lambda(d, n, k)=Q_{1}(d, n)+2 Q_{2}(d, n)+\cdots+2^{n-k-1} Q_{n-k}(d, n)+2^{n-k} \mu_{d}(k),
$$

where $Q_{i}$ is a polynomial in $d$ of degree $n-i+1$; the leading coefficient of $Q_{i}$ is a polynomial $P_{i} \in \mathbb{Q}[n]$ of degree $k-1$ and leading coefficient $(k+1) / 2$ and the other terms are polynomials in $n$ of degree $h=\max \{k-1,1\}$.

As an immediate corollary we have

Corollary 2.7. With $k$ fixed, $d>1, \lambda(d, n, k)$ is asymptotically equal to $\frac{1}{2}(k+1)$ $n^{k-1} d^{n}$ as $n$ tends to infinity.

We now have three different upper bounds for $\Phi_{d}(n, k)$, namely the one above, $\lambda(d, n, k)$, the Milnor bound, $\mu\left(d^{(n)}=d(2 d-1)^{n-1}\right.$, and the bound $d^{n+k-1}$ (remark 3 of Section A above). Depending on the values of $n, k$, and $d$, one of these bounds is better than the other two. For instance, for every fixed $k$, there exists $n_{k}$ big enough such that, for every $d \geq n \geq n_{k}$, the best bound for $\Phi_{d}(n, k)$ is $\lambda(d, n, k)$. On the contrary, if $k$ is "big" and $n-k$ "small," the best bound is $\mu_{d}^{(n)}$, or $d^{n+k-1}$, depending on the value of $d$.

The proof of Proposition 2.6 follows immediately from the lemma below about $H_{k}^{n}(d)$.

Lemma 2.8. Let $k$ be fixed. Then $H_{k}^{n}(d)$ is a polynomial of degree $n$ in the variable $d$. The leading coefficient of $H_{k}^{n}(d)$ is a monic polynomial in $n$ of degree $h=$ $\max \{y, k-1\}$, and the others coefficients are polynomials in $n$ of degree $h$.

Proof. We write $\left[X_{1}, \ldots, X_{j} \mid s_{1}, \ldots, s_{j}\right]$ to mean: "a polynomial in the variable $X_{1}, \ldots, X_{j}$ of degree $\leq s_{i}$ with respect to $X_{i}$." Then it is enough to show that

$$
\chi_{k}^{n}(d)=(-1)^{n-k}\left(n^{k-1}+[n \mid k-2]\right) d^{n}+[d, n \mid n-1, \max \{1, k-1\}]
$$


where $\chi_{k}^{n}(d)=\chi_{k}^{n}(d, \ldots, d)$. We obtain it by an easy double induction on $k$ and $n$. For $k=1, \chi_{1}^{1}(d)=d$.

$$
\begin{aligned}
\chi_{1}^{n}(d) & =d \chi_{0}^{n-1}(d)-(d-1) \chi_{1}^{n-1}(d) \\
& =n d-d(-1)^{n-2} d^{n-1}-(d-1)[d, n \mid n-2,1] \\
& =(-1)^{n-1} d^{n}+[d, n \mid n-1,1] .
\end{aligned}
$$

For $k>1$, we have

$$
\begin{aligned}
\chi_{k}^{n}(d)= & d \chi_{k-1}^{n-1}(d)-(d-1) \chi_{k}^{n-1}(d) \\
= & d(-1)^{n-k}\left(n^{k-2}+[n \mid k-3]\right) d^{n-1}+d[d, n \mid n-2, k-2] \\
& -d(-1)^{n-1-k} d^{n-1}\left(n^{k-1}+[n \mid k-2]\right)-(d-1)[d, n \mid n-2, k-1] \\
= & (-1)^{n-k} d^{n}\left(n^{k-1}+[n \mid k-2]\right)+[d, n \mid n-1, k-1] .
\end{aligned}
$$

The lemma is proved.

It remains to prove Proposition 2.5.

Proof of Proposition 2.5. It is the proof we have outlined in the introduction.

(a) As $Y \in C(d, n, k)$ is compact and $\operatorname{dim} Y \geq 1$, then there are at most $h=$ $\operatorname{dim}\left(Y \cap \mathbb{R} P^{n}\right)+1$ nonzero Betti numbers of $Y \cap \mathbb{R} P^{n}$, and the first of these numbers coincides with the last one (we are working over the field $\mathbb{Z} / 2 \mathbb{Z}$ ) and is equal to the number of connected components of $Y \cap \mathbb{R} P^{n}$. Hence it is clear that

$$
\theta_{k}^{n}(d) \leq \frac{1}{2} \sup \left\{\operatorname{Betti}\left(Y \cap \mathbb{R} P^{n}\right) \mid Y \in C(d, n, k)\right\}
$$

On the other hand, applying Smith's theory (see [B2]) - note that $Y$ is compact and $Y \cap \mathbb{R} P^{n}$ is the set of fixed points of the involution induced by the complex conjugation-we get

$$
\operatorname{Betti}\left(Y \cap \mathbb{R} P^{n}\right) \leq \operatorname{Betti}(Y)
$$

(a) is proved.

(b) Let $f \in A(d, n, k)$ and assume that $Z_{f}$ contains $s$ noncompact components $Z_{1}, \ldots, Z_{s}$. Let us denote $D(0, t)=\left\{x \in \mathbb{R}^{n} \mid\|x\|<t\right\}, S(0, t)=\{\|x\|=t\} . Z_{f}$ is "conic at infinity," that is, there exists $t_{0}$ such that $\left(\mathbb{R}^{n} \backslash D\left(0, t_{0}\right)\right) \cap Z_{f}$ is homeomorphic to $\left(Z_{f} \cap S\left(0, t_{0}\right)\right) \times\left[t_{0},+\infty[\right.$, via a homeomorphism sending $Z_{f} \cap S(0, t)$ onto $\left(Z_{f} \cap S\left(0, t_{0}\right)\right) \times\{t\}$. For every integer $m, m>t_{0}$, for every $j=1, \ldots, s$, consider a point $y_{m}^{j} \in\left(\mathbb{R}^{n} \backslash D(0, m)\right) \cap Z_{j}$, and set $x_{m}^{j}=$ $y_{m}^{j} /\left\|y_{m}^{j}\right\| \in S(0,1)=S^{n-1}$. Taking subsequences if necessary, we may assume that each $x_{m}^{j}$ converges to a point $x_{0}^{j}$ of $S^{n-1}$. It may happen that $x_{0}^{j}=x_{0}^{i}$ for $i \neq j$, hence every $x_{0}^{j}$ has a natural "multiplicity." Let $H_{0}$ be a hyperplane through the origin of $\mathbb{R}^{n}$ such that all the limit points are contained in $S^{n-1} \backslash H_{0}$. Then there exists a half-sphere $S_{+}^{n-1}$ containing at least $s / 2$ of these points (if $s$ is even) or $(s+1) / 2$ points (if $s$ is odd). If $H$ is a hyperplane parallel to $H_{0}$ and sufficiently far from $H_{0}$ in 
the direction of $S_{+}^{n-1}$ then $H$ intersects at least $s / 2$ noncompact components of $Z_{f}$, that is, $s / 2 \leq B\left(H \cap Z_{f}\right) \leq \Phi_{d}(n-1, k)$. Thus (b) is proved.

(c) Let $\nabla_{0}$ be a fixed $k$-simplex in $\mathbb{R}^{k}$. It is determined by its vertices $\nabla_{0}=$ $\nabla\left(v_{0}, \ldots, v_{k}\right)=\left\{y \in \mathbb{R}^{k} \mid y=\sum_{i} t_{i} v_{i}, t_{i} \geq 0, \sum t_{i}=1\right\}$. We assume that the origin is contained in the interior of $\nabla_{0}$. The boundary $\partial \nabla_{0}=\left\{Y \in \nabla_{0} \mid\right.$ there exists $i$ such that $\left.t_{i}=0\right\}$ is a union of "faces" of dimension $k-1$. Clearly, there are $\left(\begin{array}{c}k+1 \\ j\end{array}\right)$ faces of dimension $j-1$. Our aim is to bound $\Phi_{d}^{\mathbf{c}}(n, k)$ by means of $B^{\mathbf{c}}\left(f^{-1}\left(\partial \nabla_{0}\right)\right)$, for every $f \in A(d, n, k)$ and providing $\nabla_{0}$ is "small" and "generic" in a sense made precise later.

Let us define a function $L=L_{\nabla_{0}}: \mathbb{R}^{k} \rightarrow \mathbb{R}$ as follows. For every $t \in \mathbb{R}, t>0$, let $\nabla_{t}$ be the image of $\nabla_{0}$ by the map $y \rightarrow t y$. For every $y \in \mathbb{R}^{k}, y \neq 0$, there exists a unique $t>0$ such that $y \in \partial \nabla_{t}$. Set

$$
\begin{aligned}
& L(0)=0, \\
& L(y)=t \quad \text { (defined as above). }
\end{aligned}
$$

It is easy to see that $L$ is a continuous, positive semialgebraic function such that:

(i) $\{L=0\}=\{0\}$;

(ii) $\partial \nabla_{0}=\{L=1\}$, and, for $t>0, \partial \nabla_{t}=\{L=t\}$.

For every $f \in A(d, n, k)$, define $f_{\nabla_{0}}=\left(L_{\nabla_{0}} \circ f\right)$; it is a positive continuous semialgebraic function and its zero set coincides with $Z_{f}$.

Definition 2.9. If $\varepsilon>0$ is small enough, then every $(k+1)$-tuple $\left(w_{0}, \ldots, w_{k}\right)$, $w_{i} \in \mathbb{R}^{k}$ such that $\left\|w_{i}-v_{i}\right\|<\varepsilon$, determines a $k$-simplex $\nabla=\nabla\left(w_{0}, \ldots, w_{k}\right)$ containing the origin in its interior. We call the set of such simplexes the $\varepsilon$-neighbourhood of $\nabla_{0}$; for every $\varepsilon$ it is denoted by $W\left(\nabla_{0}, \varepsilon\right)$; for every simplex $\nabla \in W\left(\nabla_{0}, \varepsilon\right)$ we can consider the similar functions $L_{\nabla}$ and $f_{\nabla}$.

The following lemma is evident.

Lemma 2.10. For every subset $K$ of $\mathbb{R}^{n}$, for every $\varepsilon>0$, set $K_{\varepsilon}=$ $\left\{x \in \mathbb{R}^{n} \mid \operatorname{dist}(x, K) \leq \varepsilon\right\} ; \operatorname{dist}(\cdot, \cdot)$ means the Euclidean distance. Let $f \in A(d, n, k)$ and let $Z^{1}, \ldots, Z^{s}$ be the compact components of $Z_{f}$. Then there exists $\varepsilon_{0}>0$, such that, for every $i=1, \ldots, s$, for $0<\varepsilon \leq \varepsilon_{0}$ :

(i) $Z_{\varepsilon}^{i}$ is compact and connected.

(ii) $Z_{\varepsilon}^{i} \cap\left(Z_{f}-Z^{i}\right)_{\varepsilon}=\varnothing$.

Lemma 2.11. Let $\nabla_{0}, f$ be as above. Let $\varepsilon_{0}$ be as in Lemma 2.10. Then there exist $\varepsilon>0, \delta>0$ such that, for every $\nabla \in W\left(\nabla_{0}, \varepsilon\right)$ ( $\varepsilon$ is small enough), $\left\{f_{\nabla} \leq \delta\right\} \cap Z_{\varepsilon_{0}}^{i}$ is the union of certain compact connected components of $\left\{f_{\nabla} \leq \delta\right\}$, for $i=1, \ldots, s$.

Proof. The argument is quite standard (see Lemma 1 of [T], [D], and [BR]). Set $Z^{\mathrm{c}}$ to be the union of the $Z^{i}$ 's and $U$ to be the union of the $Z_{\varepsilon_{0}}^{i} . U$ is a compact semialgebraic subset of $\mathbb{R}^{n}$. Fix $\varepsilon$ as in Definition 2.9: For every $\nabla \in W\left(\nabla_{0}, \varepsilon\right)$ let us consider the restriction to $U$ of the two functions $\operatorname{dist}\left(\cdot, Z^{\mathrm{c}}\right)$ and $f_{\nabla}$. They are 
positive continuous semialgebraic functions with the same zero set equal to $Z^{\mathrm{c}}$. By the Lojasiewicz inequality we know that there exists a positive interger $m$ and a positive constant $b$ such that

$$
f_{\nabla}(x)>b \operatorname{dist}\left(x, Z^{c}\right)^{m} \quad \text { for } \quad x \in U .
$$

Choose $0<\delta^{\prime}<\min (b, \varepsilon)$. Then, for every $x \in\left\{\operatorname{dist}\left(\cdot, Z^{\mathrm{c}}\right)=\delta^{\prime}\right\}, f_{\mathrm{V}}(x)>\delta^{\prime m+1}$. If we take $\delta<\delta^{\prime m+1}$, then every component of $\left\{f_{\nabla}<\delta\right\}$ containing a component of $Z^{\mathrm{c}}$ is contained in $Z_{\delta}^{\mathrm{c}}, \subset U$. In order to finish the proof, it is enough to remark that by the proof of the Lojaswiewicz inequality (see for example, [BCR]) we can take the same $m$ for every $\nabla \in W\left(\nabla_{0}, \varepsilon\right)$ and that $b$ is a continuous function of $\varepsilon$.

Corollary 2.12. Let $F, F_{\nabla}, \varepsilon_{0}, \varepsilon, \delta$ be as in the above lemma. Then, for every $\nabla \in W\left(\nabla_{0}, \varepsilon\right), B^{\mathrm{c}}\left(\left\{f_{\nabla}=\delta\right\}\right)=B^{\mathfrak{c}}\left(f^{-1}\left(\partial \nabla_{\delta}\right)\right)$.

Let $\nabla$ be as before. For every face $\sigma$ of dimension $j$ of $\nabla$, let us consider the affine subspace $H_{\sigma}$ of $\mathbb{R}^{k}$ spanned by $\sigma$. Let us denote by $C$ the critical values set of the map $f$. It is known that $C$ is a semialgebraic set in $\mathbb{R}^{k}$ of dimension $<k$. This follows by the semialgebraic version of the Morse-Sard lemma which is actually much more easily proved. Fix a Whitney stratification of $C$ made by a finite union of analytic, semialgebraic submanifolds of $\mathbb{R}^{k} . C=\bigcup M_{i}, i=1, \ldots, h$.

Definition 2.13. We say that $\nabla$ is in general position with respect to $f$ if, for every face $\sigma$ of $\nabla, H_{\sigma}$ is transverse to every stratum $M_{i}$ of the stratification of $C$.

The following lemma is a consequence of standard transversality arguments.

Lemma 2.14. The set of $\nabla$ 's in $W\left(\nabla_{0}, \varepsilon\right)$ in general position with respect to $f$ is an open dense subset.

Then, using the last lemma and Corollary 2.12, it is clear that, in order to bound $\Phi_{d}^{\mathrm{c}}(n, k)$ and finish the proof of Proposition 2.5, it is enough to bound $B^{\mathrm{c}}\left(f^{-1}(\partial \nabla)\right)$ for any $\nabla$ in general position with respect to $f$. From now on $\nabla$ is assumed in general position. For $i=0,1, \ldots, k-1, \nabla_{i}$ denotes the $i$ th skeleton of $\nabla$.

Definition 2.15. For every $i$-dimensional face $\sigma \subset \nabla_{i}$, we say that a compact connected component $T$ of $f^{-1}(\sigma)$ is pure if $T \cap f^{-1}\left(\nabla_{i-1}\right)=\varnothing$.

Let $h_{i}$ be the number of connected components of $f^{-1}\left(\nabla_{i}\right)$ which are compact and pure for some $\sigma$, face of dimension $i$.

Lemma 2.16. $\quad B^{\mathrm{c}}\left(f^{-1}(\partial \nabla)\right) \leq \sum_{0 \leqq j \leqq k-1} h_{j}$.

Proof. By induction on $i$, that is, for $i=0, \ldots, k-1$,

$$
B^{c}\left(f^{-1}\left(\nabla_{i}\right)\right) \leqq \sum_{0 \leqq j \leqq i} h_{j}
$$


Evidently $B^{\mathrm{c}}\left(f^{-1}\left(\nabla_{0}\right)\right)=h_{0}$. The number of compact components $\Gamma$ of $f^{-1}\left(\nabla_{i}\right)$ such that $\Gamma \cap f^{-1}\left(\nabla_{i-1}\right) \neq \varnothing$ is clearly smaller than $B^{\mathbf{c}}\left(f^{-1}\left(\nabla_{i-1}\right)\right)$, and so $B^{\mathrm{c}}\left(f^{-1}\left(\nabla_{i}\right)\right) \leqq B^{\mathrm{c}}\left(f^{-1}\left(\nabla_{i-1}\right)\right)+h_{i}$.

Lemma 2.17. For every $i=0, \ldots, k-1$

$$
h_{i} \leqq\left(\begin{array}{c}
k+1 \\
i+1
\end{array}\right) \theta_{k+1-(i+1)}^{n}(d) .
$$

Proof. Consider $\sigma \in \nabla_{i}, \operatorname{dim} \sigma=i$. As before consider $H_{\sigma}$. The pure components in $f^{-1}(\sigma)$ are, in particular, compact components of $S=f^{-1}\left(H_{\sigma}\right)$. If $H_{\sigma}$ is defined by the equations of degree $1, r_{1}\left(y_{1}, \ldots, y_{k}\right)=\cdots=r_{k-i}\left(y_{1}, \ldots, y_{k}\right)=0$, then they are compact components of $S=\{r f=0\}, \quad r=\left(r_{1}, \ldots, r_{k-i}\right)$. Note that $r f \in A(d, n, k-i)$. Since $\nabla$ is in general position, $S$ is a nonsingular algebraic set in $\mathbb{R}^{n}$ of codimension $n-i$. Let us consider the projective closure $S^{\prime}$ of $S$ in $\mathbb{R} P^{n}$. By a weak application of the Bertini theorem [GWDL] there is a complex complete intersection $Y \in C(d, n, k)$ such that the equations of $Y \cap \mathbb{R} P^{n}$ are close to the equations of $S^{\prime}$. Then every compact component of $S$ is approximated by one component of $Y \cap \mathbb{R} P^{n}$. The lemma is now proved simply noting that there are $\left(\begin{array}{c}k+1 \\ i+1\end{array}\right)$ faces of dimension $i$ in $\nabla_{i}$.

This conclude the proof of Proposition 2.5.

\section{Remarks and Complements}

1. It could seem a little redundant to bound only the number of connected components in terms of the sum of all Betti numbers (as in the proof of (a) of Proposition 2.5). But it is not so redundant: actually it may happen that $Y \cap \mathbb{R} P^{n}$ is a union of spheres, in such a case

$$
2 B\left(Y \cap \mathbb{R} P^{n}\right)=\operatorname{Betti}\left(Y \cap \mathbb{R} P^{n}\right)
$$

2. With slight modifications to the method used above, we can obtain other bounds in terms of $(d, \mathrm{n}, \mathrm{k})$ which are asymptotically worse than $\lambda(d, n, k)$ (for $k$ fixed and $n$ tending to infinity), but presumably better for some intermediary values of $n$ (in the spirit of remark 3 of Section A). For example, it is clear from the proof of (b) of Proposition 2.5, that

$$
\Phi_{d}(n, k) \leqq \Phi_{d}^{\mathrm{c}}(n, k)+\Phi_{d}^{\mathrm{c}}(n, k+1) .
$$

Then we can bound the last term by applying (c) of proposition 2.5 twice.

Another variation is the following. 
Lemma 2.18. For every positive integer $i$, there exist $a k$-simplex $\nabla^{i}$ and $a$ $(k-1)$-face $F^{i}$ of $\nabla^{i}$ such that:

(a) $\operatorname{dist}\left(0, \partial \nabla^{i}\right) \leqq 1 / i$;

(b) $\nabla^{i}$ is in general position with respect to $f$;

(c) $\#\left\{j \mid f^{-1}\left(F^{i}\right) \cap Z_{\varepsilon_{0}}^{j} \neq \varnothing\right\} \geqq s / 2$.

(We have used the notations introduced during the proof of Proposition 2.5.)

Proof. The proof of (a) and (b) is essentially done in the previous discussion. In order to prove (c), we work as in the proof of (b) of Proposition 2.5 but at the origin instead of at infinity.

For every $j=1, \ldots, s, f\left(Z_{\varepsilon_{0}}^{j}\right)$ is a connected semialgebraic set in $\mathbb{R}^{k}$ of dimension $\geqq 1$ and containing the origin. By the "curve selection lemma" (see [M2] or $[\mathrm{BCR}])$ there exists a germ at the origin of $\mathbb{R}^{k}$ of an analytic arc $\gamma_{j} \subset f\left(z_{\varepsilon_{0}}^{j}\right)$. Then we can choose a hyperplane $H$ intersecting at least half of the rays tangent to all $\gamma_{j}$ 's at the origin. If $H_{i}$ is parallel to $H$ and sufficiently close to 0 , then $H_{i} \cap \gamma_{j} \cap$ $\{\|y\|<1 / i\} \neq \varnothing$ for at least $s / 2$ of the $\gamma_{j}$ 's. It is now easy to conclude the proof of the lemma.

Using the last lemma we obtain the following version of Proposition 2.5.

\section{Proposition 2.5'}

$\left(\mathrm{b}^{\prime}\right) \Phi_{d}(n, k) \leqq \Phi_{d}^{\mathrm{c}}(n, k)+2 \Phi_{d}(n-1, k)$.

(c') $\Phi_{d}^{c}(n, k) \leqq 2 \sum_{1 \leqq j \leqq k}\left(\begin{array}{l}k \\ j\end{array}\right) \Phi_{k+1-j}^{n}(d)$.

Using Proposition (2.5.) it is clear how to define recursively another bound for $\Phi_{d}(n, k)$.

3. The bound obtained in the previous section (i.e., $\lambda(d, n, k)$ ) could also be used to improve known bounds for the number of connected components of further semialgebraic sets. For example, using the method of Warren [W, Theorem 2] we get, for every $f \in A(d, n, k)$,

$$
B\left(\mathbb{R}^{n}-Z_{f}\right) \leqq \sum_{0 \leqq j \leqq n} 2^{j} C_{k, j} \lambda(d, n, j),
$$

where $C_{k, j}$ is the usual binomial coefficient, except that $C_{k, j}=0$ for $k<j$.

This concludes our discussion about the bound for $B\left(Z_{f}\right)$ in terms of the "measure of complexity" of $f$ given by $(d, n, k)$.

\section{Bounding $B\left(Z_{f}\right)$ Using Newton Polyhedra}

We need some preliminary definitions. Let $f \in \mathbb{C}\left[X_{1}, \ldots, X_{n}\right], f=\sum c_{q} X^{q}, q=$ $\left(q_{1}, \ldots, q_{n}\right) \in \mathbb{N}^{n}$, and $X^{q}=X_{1}^{q_{1}} X_{2}^{q_{2}} \ldots X_{n}^{q_{n}}$. The support of $f$ is the set of $q$ such that $c_{q} \neq 0$. It can be considered as a finite subset of the standard lattice $\mathbb{Z}^{n}$ contained in $\mathbb{R}^{n}$. The Newton polyhedron of $f$, denoted $\Delta_{f}$, is the convex hull in $\mathbb{R}^{n}$ of the union 
of its support and $\{0\}$. If $C_{1}$ and $C_{2}$ are convex integral polyhedra in $\mathbb{R}^{n}$ (i.e., with vertices in the lattice $\mathbb{Z}^{n}$ ), then

$$
C_{1}+C_{2}:=\left\{q_{1}+q_{2} ; q_{1} \in C_{1}, q_{2} \in C_{2}\right\}
$$

is also a convex integral polyhedron.

Let $C_{1}, \ldots, C_{n}$ be convex (integral) polyhedra in $\mathbb{R}^{n}$; we define the mixed volume of $C_{1}, \ldots, C_{n}$ by the formula

$$
\begin{aligned}
& V\left(C_{1}, \ldots, C_{n}\right) \\
& =1 / n !\left[(-1)^{n-1} \sum_{1 \leqq i \leqq n} V_{n}\left(C_{i}\right)+(-1)^{n-2} \sum_{1 \leqq i<j \leqq n} V_{n}\left(C_{i}+C_{j}\right)\right. \\
& \left.\quad+\cdots+V_{n}\left(C_{1}+\cdots+C_{n}\right)\right]
\end{aligned}
$$

where $V_{n}(C)$ denotes the volume of any convex polyhedron $C$ in $\mathbb{R}^{n}$, normalized by requiring that the parallelepiped of the lattice has volume equal to 1 . With this definition, we have $V(C, \ldots, C)=V_{n}(C)$, for any convex polyhedron $C$ in $\mathbb{R}^{n}$.

If $F$ is a monomial of degree $n$ in the $k$ variables $X_{1}, \ldots, X_{k}, F=X_{1}^{n_{1}} X_{2}^{n_{2}} \cdots X_{k}^{n_{k}}$, we set

$$
F\left(C_{1}, \ldots, C_{k}\right)=n ! V\left(C_{1}, \ldots, C_{1}, \ldots, C_{k}, \ldots, C_{k}\right)
$$

where $C_{i}$ is taken $n_{i}$-times. If $F$ is an analytic function defined near 0 in the variable $X_{1}, \ldots, X_{k}$ we set

$$
F\left(C_{1}, \ldots, C_{k}\right)=F_{n}\left(C_{1}, \ldots, C_{k}\right)
$$

where $F_{n}$ is the homogeneous component of degree $n$ in the Taylor series of $F$ at 0 , and the above definition is extended by linearity.

Let us now consider a polynomial function $g=\left(g_{1}, \ldots, g_{k}\right): \mathbb{C}^{n} \rightarrow \mathbb{C}^{k}$. Let us denote by $\Delta_{1}, \ldots, \Delta_{k}$ the Newton polyhedra of $g_{1}, \ldots, g_{k}$. We denote by $Z_{g}(\mathbb{C})$ its complex zero set. For every subset $I \subset\{1, \ldots, n\}$, set $R_{I}=\left\{x_{i}=0\right.$ for $\left.i \in I\right\} \subset \mathbb{R}^{n}$. Set $\Delta_{i}^{I}=\mathbb{R}_{I} \cap \Delta_{i}, \Delta_{i}^{\varnothing}=\Delta_{i}$. The number $\prod_{1 \leqq i \leqq k} \Delta_{i}^{I}\left(1+\Delta_{i}^{i}\right)^{-1}$ is obtained by substituting the polyhedra $\Delta_{i}^{I}$ into the analytic function $\prod_{1 \leqq i \leqq k} x_{i}\left(1+x_{i}\right)^{-1}$ in the way we have explained above.

The result of Hovansky which we use is the following.

Proposition 3.1 (see the theorem of Section 4 of [H2]). Assume that $g_{1}, \ldots, g_{k}$ are "nondegenerate for the Newton polyhedra" and that every support of these polynomials contains the origin. Then the Euler characteristic of $Z_{g}(\mathbb{C})$ is given by the formula

$$
E\left(Z_{g}(\mathbb{C})\right)=\sum_{I} \prod_{1 \leqq i \leqq k} \Delta_{i}^{I}\left(1+\Delta_{i}^{I}\right)^{-1}
$$


Remark 3.2. The notion of "nondegenerate polynomial for the Newton polyhedron" is a little too technical to be recalled here (see [H1] and [H2]). What is important for us is that:

(a) If $g_{1}, \ldots, g_{k}$ (as in the above proposition) are nondegenerate for the Newton polyhedron and with supports containing the origin, then $Z_{g}(\mathbb{C})$ is a smooth complex complete intersection of complex dimension $n-k$ in $\mathbb{C}^{n}$.

(b) The $k$-tuples of real polynomials (that is with real coefficients) nondegenerate for the Newton polyhedron are generic and hence dense, for the natural topology on the coefficients, in the set of all $k$-tuples of polynomials with the same given Newton polyhedra (see the theorem of paragraph 2 of [H1]).

In fact, as in Section 2, we are interested in the sum of Betti numbers over $\mathbb{Z} / 2 \mathbb{Z}$ of $Z_{g}(\mathbb{C})$, Betti $\left(Z_{g}(\mathbb{C})\right.$ ). We deduce it from Remark 3.2 using a further technical condition.

Definition 3.3. We say that $g=\left(g^{1}, \ldots, g_{k}\right)$ satisfies condition (*) if for each nonempty $K \subset\{1, \ldots, k\}$ the dimension of the polyhedron $\sum_{i \in K} \Delta_{i}$ is at least $n-k+\#(K)$. Note that $(*)$ is actually a condition on the Newton polyhedra.

Remark 3.4. If, for example, every $\Delta_{i}$ has dimension $n$, then $g$ satisfies condition (*).

Lemma 3.5. If $g$ satisfies the hypothesis of Proposition 3.1 and also condition (*), then

$$
\operatorname{Betti}\left(Z_{g}(\mathbb{C})\right)=\left(1+(-1)^{n-k-1}\right)+(-1)^{n-k} E\left(Z_{g}(\mathbb{C})\right)
$$

Proof. It is an immediate consequence of the following fact: under the hypotheses of the lemma we have that the $i$ th Betti number of $Z_{g}(\mathbb{C}), b_{i}\left(Z_{g}(\mathbb{C})\right)=0$ for $i \neq 0$, $n-k$. This is easily deduced from Corollary 3.8 of $[\mathrm{DH}]$ by using the construction in Section 6.2 of [DH]: we use the hypersurface with equation

$$
G(t, x)=\sum_{1 \leqq i \leqq k} t_{i} g_{i}(x)-1=0
$$

The hypotheses guarantee that $G(t, x)$ is nondegenerate for its Newton polyhedron and that the polyhedron is of dimension $n+k$.

Let us fix $k$ integral polyhedra in the positive quadrant $\mathbb{R}_{+}^{n}: \Delta=\left(\Delta_{1}, \ldots, \Delta_{k}\right)$. We assume that every $\Delta_{i}$ contains the origin. Let us denote by $A(\Delta)$ the set of polynomial maps $f=\left(f_{1}, \ldots, f_{k}\right): \mathbb{R}^{n} \rightarrow \mathbb{R}^{k}$ such that, for every $i, \Delta_{i}$ is the Newton polyhedron of $f_{i}$. We want to show that, for every $f \in A(\Delta), B\left(Z_{f}\right)$ can be bounded in terms of $\Delta$. 
Fix some further notations:

$$
\begin{aligned}
\Phi(\Delta) & =\sup \left\{B\left(Z_{f}\right) \mid f \in A(\Delta)\right\} \\
\Phi^{\mathrm{c}}(\Delta) & =\sup \left\{B^{\mathrm{c}}\left(Z_{f}\right) \mid f \in A(\Delta)\right\} \\
(\Delta) & =\sup \left\{B\left(Z_{g}\right) \mid g \in A(\Delta) \text { and } g\right. \text { regarded as a complex polynomial } \\
& \text { map satisfies the hypotheses of Lemma } 3.5\}
\end{aligned}
$$

For $i, 1 \leqq i \leqq n$, set

$$
\pi_{i}(\Delta)=\left(\pi_{i}\left(\Delta_{1}\right), \ldots, \pi_{i}\left(\Delta_{k}\right)\right)
$$

where $\pi_{i}$ denotes the natural projection onto the plane $\left\{x_{i}=0\right\}$. For $1 \leq j \leq k$ set

$$
r_{j}(\Delta)=\left(\Delta_{1}, \ldots, \Delta_{i-1}, \Delta_{i+1}, \ldots, \Delta_{k}\right) .
$$

For every $J \subset\{1, \ldots, k\}, J=\left\{j_{1} \leq \ldots \leq j_{s}\right\}, s<k$, set

$$
r_{j}(\Delta)=r_{j_{1}} r_{J-\left\{j_{1}\right\}}(\Delta)
$$

We are now able to state a proposition, similar to Proposition 2.5, which actually contains the recursive definition of an upper bound for $\Phi(\Delta)$.

Proposition 3.6. If $\Delta$ satisfies condition (*), then

$$
\begin{aligned}
& \text { (a) } \quad \Phi(\Delta) \leq\left(1+(-1)^{n-k-1}\right)+(-1)^{n-k}\left(\sum_{I, 1 \leq i \leq k} \Delta_{i}^{I}\left(1+\Delta_{i}^{I}\right)^{-1}\right) \\
& \text { (b) } \quad \Phi(\Delta) \leq \Phi^{\mathrm{c}}(\Delta)+2\left(\sum_{1 \leq i \leq n} \Phi\left(\pi_{i}(\Delta)\right)\right) \\
& \text { (c) } \quad \Phi^{\mathrm{c}}(\Delta) \leq \sum_{\substack{j \subset\{1, \ldots, k\} \\
J \neq\{\ldots, k\}}} 2^{k-\#\{J\}} \theta\left(r_{J}(\Delta)\right) .
\end{aligned}
$$

Proof. The proof is similar to the one of Proposition 2.5. So we only note the main changes.

(a) Use again Smith's theory, Proposition 3.1, and Lemma 3.5.

(b) If $H$ is an arbitrary hyperplane in $\mathbb{R}^{n}$, then we have no control of the Newton polyhedra of the polynomials defining $Z_{f} \cap H$. So we have to change the argument a little. For every $i=1, \ldots, n$ let $s_{i}$ be the number of limit points in $S^{n-1}-\left\{x_{i}=0\right\}$ (see the proof of Proposition 2.5(b)). They are distributed in the two connected components of $S^{n-1}-\left\{x_{i}=0\right\}: s_{i}^{\prime}$ in one component and $s_{i}^{\prime \prime}$ in the other. So that we can write $s_{i}=s_{i}^{\prime}+s_{i}^{\prime \prime}$ and assume $s_{i}^{\prime} \geq s_{i}^{\prime \prime}$. It is clear that

$$
\sum_{1 \leq i \leq n} s_{i}^{\prime} \geq s / 2
$$


If $H_{i}=\left\{x_{i}=c_{i}\right\}, i=1, \ldots, n$, are affine hyperplanes with $\left\|c_{i}\right\|$ big enough and the sign of $c_{i}$ suitably chosen, then

$$
s / 2 \leq \sum_{1 \leq i \leq n} s_{i}^{\prime} \leq \sum_{1 \leq i \leq n} B\left(Z_{f} \cap H_{i}\right)
$$

To conclude it is enough to remark that $Z_{f} \cap H_{i}$ is defined by $k$ equations in the variables $X_{1}, \ldots, X_{i-1}, X_{i+1}, \ldots, X_{n}$ belonging to $A\left(\pi_{i}(\mathbf{\Lambda})\right)$.

(c) Let us remark first that if $\Delta$ satisfies condition (*), then each $\pi_{i}(\Delta)$ and $r_{J}(\Delta)$ also satisfy this condition. The proof is essentially the same as in (c) of Proposition 2.5. We simply replace the small generic simplex with a small generic parallelepiped $P$ in $\mathbb{R}^{k}$ with the faces parallel to the coordinate planes, that is $P$ may be defined as

$$
\begin{aligned}
P=\left\{y_{1} \leqq c_{1}\right\} \cap\left\{y_{1} \geqq d_{1}\right\} \cap \cdots \cap\left\{y_{k} \leqq c_{k}\right\} \cap\left\{y_{k} \geqq d_{k}\right\}, \\
c_{i}>0, \quad d_{i}<0 \text { for } i=1, \ldots, k
\end{aligned}
$$

Consider the set of all faces of $P$ of dimension $<k$. If $\sigma$ is such a face $H_{\sigma}$ denotes, as usual, the affine plane spanned by $\sigma$. As for the proof of Proposition 2.5 call $C \subset R^{k}$ the critical value set of any fixed $f \in A(\Delta)$. Fix a Whitney stratification of $C$ and let us say $P$ in general position with respect to $f$ if all $H_{\sigma}$ as above are transverse to every stratum of the stratification of $C$. Again the set of $P$ in general position is a dense set (in the suitable space of parallelepipeds) and if $P$ is small enough, then $B^{\mathrm{c}}\left(f^{-1}(\partial P)\right) \geqq B^{\mathrm{c}}\left(Z_{f}\right)$ (see the details of the proof of Proposition 2.5). Thus in order to bound $B^{\mathrm{c}}\left(Z_{f}\right)$ it is enough to bound $B^{\mathrm{c}}\left(f^{-1}(\partial P)\right)$ for every $P$ in general position with respect to $f$. For every $i=0, \ldots, k-1$, let $P_{i}$ denote the ith-skeleton of $P$. For every face $\sigma$ of dimension $r$ in $\partial P$ there exists $J_{\sigma} \subset\{1, \ldots, k\} \quad\left(J_{\sigma} \neq\{1, \ldots, k\}\right)$ such that $\#\left\{J_{\sigma}\right\}=r$ and $\sigma$ is parallel to $R_{J_{\sigma}}=\left\{y_{j}=0 \mid j \in\{1, \ldots, k\}-J_{\sigma}\right\} \subset \mathbb{R}^{k}$. For every such subset $J$ of $\{1, \ldots, k\}$, there are $2^{k-\#\{J\}}$ corresponding faces of $\partial P$ of dimension \#\{J\} such that, for each such face $\sigma, J=J_{\sigma}$.

For every face $\sigma$ of dimension $r$ we say that a compact component $T$ of $f^{-1}(\sigma)$ is pure if $T \cap f^{-1}\left(P_{r-1}\right)=\varnothing$. Let $h_{r}$ be the number of connected components of $f^{-1}\left(P_{r}\right)$ which are compact and pure for some face $\sigma$ of dimension $r$. As in Lemma 2.16. we deduce that

$$
B^{\mathbf{c}}\left(f^{-1}(\partial P) \leqq \sum_{0 \leqq r \leqq k-1} h_{r}\right.
$$

This last fact implies that

$$
B^{\mathfrak{c}}\left(f^{-1}(\partial P)\right) \leqq \sum_{\sigma \subset \partial P} B^{\mathfrak{c}}\left(f^{-1}\left(H_{\sigma}\right)\right)
$$

Then the proof of (c) will be complete after the following lemma. 
Lemma 3.7. $B^{\mathrm{c}}\left(f^{-1}\left(H_{\sigma}\right)\right) \leqq \theta\left(r_{J_{\sigma}}(\Delta)\right)$.

Proof. $f^{-1}\left(H_{\sigma}\right)$ is defined by $k-\#\left\{J_{\sigma}\right\}$ polynomial equations and the corresponding set of Newton polyhedra is $r_{J_{\sigma}}$. To prove the lemma it is enough to consider polynomials $b_{1}, \ldots, b_{k-\#\left\{J_{\alpha}\right\}}$ such that:

(i) They have the same Newton polyhedra $r_{J_{\sigma}}(\Delta)$ and are nondegenerate for the Newton polyhedra (see Proposition 3.1 and Remark 3.2).

(ii) They are close enough to the polynomials defining $f^{-1}\left(H_{\sigma}\right)$ so that

$$
B^{\mathrm{c}}\left(f^{-1}\left(H_{\sigma}\right)\right) \leqq B^{\mathrm{c}}\left(Z_{b}\right)
$$

$$
b=\left(b_{i}\right)
$$

The existence of such $b_{i}$ 's is assured by Remark 3.2. Moreover, property (*) is preserved by the operation $r_{J_{\sigma}}$. Then the lemma is proved.

Thus (c) is also completely proved as well as Proposition 3.6.

As we said this proposition contains the recursive definition of an upper bound for $\Phi(\Delta)$ (under the technical condition $(*)$ which is in fact mild enough; see Remark 3.4). We denote such a bound by $\tau(\Delta)$.

\section{Final Remarks and Examples}

We want to compute and compare the bounds $\lambda(d, n, k)$ and $\tau(\Delta)$ in some simple examples.

(A) Let $f: \mathbb{R}^{2} \rightarrow \mathbb{R}$ have a Newton polyhedron of the form

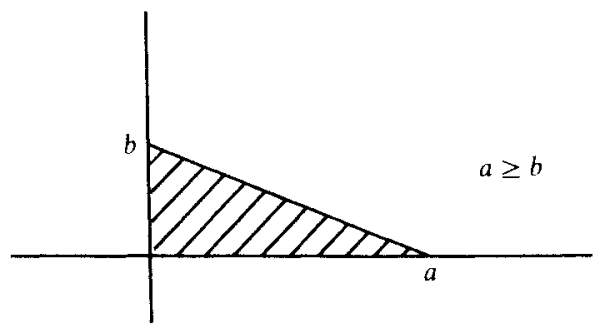

In this case $\tau$ is $2 a b+4$ and $\lambda$ is $a^{2}-a+4$.

(B) Let, more generally, $f: \mathbb{R}^{n} \rightarrow \mathbb{R}$ have a simplicial Newton polyhedron with vertices $0,\left(a_{1}, 0, \ldots, 0\right), \ldots,\left(0, \ldots, 0, a_{n}\right)$. Then

$$
\tau=2 a_{1} a_{2} \cdots a_{n}+K
$$


where $K$ means a sum of monomials of smaller degree, and

$$
\lambda=a_{1}^{n}+\text { (monomials of smaller degree) }
$$

assuming $a_{1} \geqq a_{2} \geqq \cdots \geqq a_{n}$.

(C) If the Newton polyhedron of $f$ is

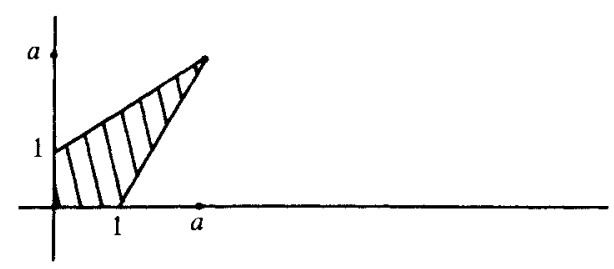

then $\tau=8 a$.

(D) If $f=\left(f_{1}, f_{2}\right): \mathbb{R}^{2} \rightarrow \mathbb{R}^{2}$ has Newton polyhedra $\Delta_{1}, \Delta_{2}$ :
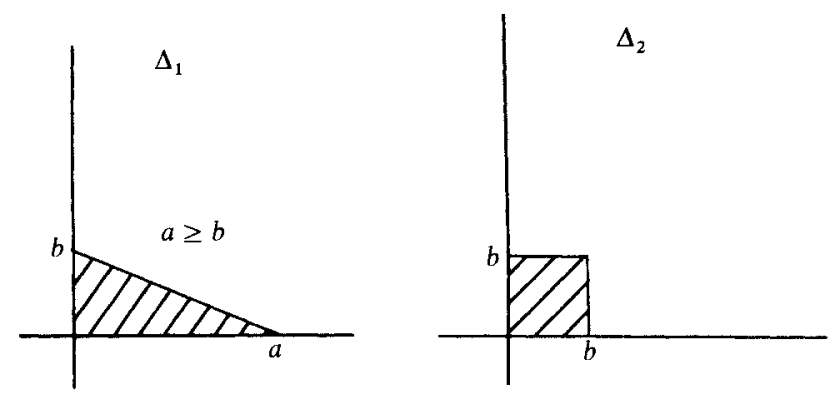

then

$$
\begin{aligned}
& \tau=2 a b-b^{2}+\text { (monomials of smaller degree), } \\
& \lambda=a^{2}+\text { (monomials of smaller degree). }
\end{aligned}
$$

It is clear from this examples that if the Newton polyhedra have some special form, then $\tau$ is better than $\lambda$.

\section{References}

[BR] Benedetti, R., and Risler J. J., Real Algebraic and Semi-Algebraic Set, Hermann, Paris, 1990.

[B1] Ben-Or, M., Lower bounds for algebraic computation trees, Proc. 15th ACM Symp. on Theory of Computing, pp. 80-86, 1983.

[BCR] Bochnak, J., Coste, M., and Roy, M. F., Géometrie algébrique réelle, Springer-Verlag, Berlin, 1987.

[B2] Bredon, G. F., Introduction to Compact Transformation Groups, Pure and Applied Mathematics, Vol. 46, Academic Press, New York, 1972. 
[DH] Danilov, V. I., and Hovansky, A. G., Newton polyhedra and an algorithm for computing Hodge Deligne numbers, Math. USSR-Izv., 29, 279 298, 1987.

[D] Durfee, A. H., Neighbourhoods of algebraic sets, Trans. Amer. Math. Soc., 276, 517-530, 1983.

[F] Fary, I., Cohomologie des varietes algébriques, Ann. of Math., 65, 21-73, 1957.

[GWDL] Gibson, C. G., Wirthmuller, K., du Plessis, A. A., and Looijenga, E. J. N, Topological Stability of Smooth Mappings, Lectures Notes in Mathematics, Vol. 552, Springer-Verlag, Berlin, 1976.

[GP] Goodman, J. E, and Pollack, R., Upper bounds for configurations and polytopes in $\mathbb{P}^{d}$, Discrete Comput. Geom., 1, 219-227, 1986.

[H1] Hovansky, A. G., Newton polyhedra and toric varieties, Functional Anal. Appl., 11, 289-296, 1977.

[H2] Hovansky, A. G., Newton polyhedra and the genus of complete intersections, Functional Anal. Appl., 12, 38-46, 1978.

[M1] Milnor, J., On the Betti numbers of real varieties, Proc. Amer. Math. Soc., 15, 275-280, 1964.

[M2] Milnor, J., Singular Points of Complex Hypersurfaces, Annals of Mathematical Studies, Vol. 61, Princeton University Press, Princeton, NJ, 1968.

[R1] Risier, J. J., Complexité et géometrie réelle, sem. Bourbaki, Asterisque, 133/134, 89-100, 1986.

[R2] Rokhlin. V. A., Congruences mod 16 in Hilbert's 16th problem, Functional Anal. Appl., 6, $301 \cdots 306,1972$.

[T] Thom, R., Sur l'homologie des varietes algébriques réelles, in Differential and Combinatorical Topology, pp. 255-265 (Cairns, S., ed), Princeton University Press, Princeton, NJ, 1965.

[W] Warren, E. H., Lower bounds for approximation by nonlinear manifolds, Trans. Amer. Math Soc., 133, 167-178, 1968.

Received September 13, 1988, and in revised form March 29, 1989 and May 29, 1990. 